\title{
A Look at Triple Arthrodesis Joint Fusion without Rigid Internal Fixation
}

\author{
Luis E. Marin, Federico A. Auger, Joanne N. Balkaran, Liliana M. Heath, Giselle Ojeda \\ Marin Foot and Ankle Center, Hialeah, USA \\ Email: drluismarin@hotmail.com
}

Received November 9, 2011; revised April 17, 2012; accepted April 26, 2012

\begin{abstract}
Triple arthrodesis is an invasive procedure that involves denuding and fusion of three foot joints; Talocalcaneal (T-C), Talonavicular (T-N) and Calcaneocuboid (C-C). Traditionally, with external fixation triple arthrodesis has been performed without corresponding internal fixation. The purpose of this study is to take a scientific look at the compressive forces acting on the joint surfaces using the arch wire technique without the use of rigid internal fixation. Five fresh cadaveric lower-limb specimens were prepared for the triple arthodesis by anatomic dissection. External fixators were applied to the limbs and pressure sensors from the Tekscan $6900 \mathrm{C}$ were placed in the three foot joints where tension was applied. After several trials with different variables it was seen that the arched wire technique on the footplate alone does not allow constant compression at the three joints. However, weightbearing increased compression across all three joints regardless of the arch wire compression. This study proves that weightbearing alone provides more compression at all 3 joints in a triple arthrodesis.
\end{abstract}

Keywords: Triple Arthrodesis; Arched Wire; External Fixation; Posterior Tibialis Tendon Dysfunction; Charcot Foot

\section{Introduction}

The triple arthrodesis is an invasive, joint destructive surgery that is used as an end-stage or salvage procedure in most cases. It involves denuding and fusion of the three joints-talonavicular (T-N), calcaneocubiod (C-C) and talocalcaneal joints (T-C). Its main indications include stage 3 PTTD (Posterior Tibialis Tendon Dysfunction), rigid pesplanovalgus, severe degenerative joint disease, post-traumatic arthritis, rheumatoid arthritis, deformity correction and charcot reconstruction.

In 1923, Ryerson described the first documented triple arthrodesis. He used no internal fixation and if there was joint instability, chromic catgut was tunneled through drill holes in order to bind the bones together [1]. Traditionally, triple arthrodeses has primarily been performed with internal fixation, which has been stabilized with application of a splint and then a period of non-weight bearing status. Internal fixation with partially threaded screws, plates or staples provide rigid compression at the three joints. Recently, triple arthrodeses have been fixated with various types of internal and/or external fixation. External fixation in combination with and without internal fixation is also used, reducing the period of non-weight bearing status.

In 1978, El Ghawabi also documented triple arthrodesis using solely an external compression "device" which was applied to secure rigid fixation of the tarsal bones. His article looked at the dimensional planes in which the three joints are orientated and the "device" distributed the built-in compression forces over their surfaces equally. His external fixation system provided centripetal compression using forces across the three differently orientated joints [2]. However, this external fixator device did not allow for weight bearing.

In 2004, Talarico and Vito described the triple arthrodesis using external ring fixation with arched-wire compression [3]. The frame consisted of two rings and a footplate. This technique involves placing a smooth wire across the navicular and cuboid bones and arching the wire posteriorly. It is then secured to the foot plate and tensioned. This theoretically allows for compression across the fusion site of the calcaneocuboid and talonavicular. Another smooth wire is driven from medial to lateral through the talus just antero-inferior to both the medial and lateral malleolus. It is deflected inferiorly and posteriorly, attached to an appropriately sized post, secured to the foot plate and tensioned. This theoretically allows for compression across the subtalar joint. The article stated that by modifying or arching the attachment of these two transosseous wires to the fixator, rigid and constant compression is produced across the three joints [3]. Patients were encouraged to weight bear on postoperative day one and an external bone stimulator was provided to each patient. In their study, 84 of 87 (97\%) patients achieved 
radiologic fusion by the sixth week. According to previous research documented for triple arthrodesis with only internal fixation, fusion time is stated to occur in 10 - 14 weeks [4]. Therefore, this study of performing triple ar throdesis with only external fixation indicated an increase in fusion time compared to triple arthrodeses with only internal fixation.

\section{Rationale}

The 2004 study was the only detailed article showing arched-wire compression technique and has been adopted by many foot and ankle surgeons in conjunction with internal fixation. The actual level of compression across the three joints using arched-wire technique has not been examined. Our research was conducted on cadaveric limb specimens using the Tekscan 6900CR pressure sensors in Model EH-1 USB handles (Tekscan, Inc. South Boston, MA) (Figure 1), and the MTS 858 MiniBionix II testing system (Figure 2). Triple arthrodesis with external fixation was performed on five cadaveric lower extremity limb specimens. The Tekscan 6900CR Pressure sensors were placed in the three joints as the transosseous wires were tensioned to measure the actual forces applied to the joints, as indicated in the 2004 study. These sensors were calibrated and placed at a medium sensitivity setting. The cadaver limb with the external fixator and pressure sensors were then placed on the MTS $858 \mathrm{MiniBi}-$ onix II testing system to simulate ambulation of a $75 \mathrm{~kg}$ person. It applies axial forces from the knee within multiple cyclic periods.

\section{Purpose}

The purpose of this study is to take a scientific look at the compressive forces acting on the joint surfaces of the talonavicular, calcaneocubiod, and talocalcaneal joints when using the arched-wire technique.

\section{Materials and Methods}

\subsection{Specimen Preparation}

Five fresh cadaveric lower-limb specimens were disarticulated at the knee joint and prepared for a triple arthrodesis by anatomic dissection and resection of the talonavicular, calcaneocuboid, and talocalcaneal joints. Four specimens were used in Trial 1 and one in Trial 2. The two incisions technique, one on the medial and one on the lateral aspect as described by Ryerson was used to gain access to the joints. The joints were identified and the cartilaginous surfaces were denuded. A saggital saw was used to reciprocal plane the joints for adequate bony coaptation (Figures 3 and $\mathbf{4}$ ).

Next, a circular ring fixator was introduced onto the field. It consisted of one tibial ring, one ankle ring and one foot-plate. Two smooth wires were consecutively

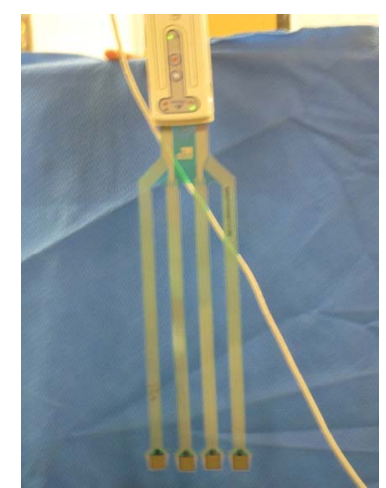

Figure 1. Tekscan 6900CR pressure sensors in Model EH-1 USB handles.

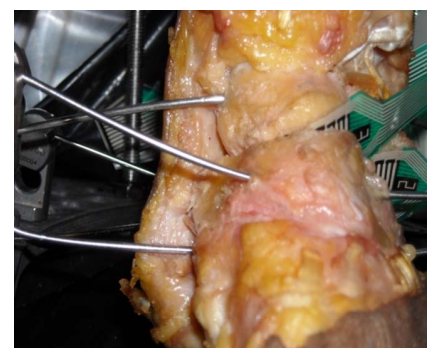

Figure 2. Demonstration of MTS 858 MiniBionix 11 on non-weightbearing cadaveric limb.

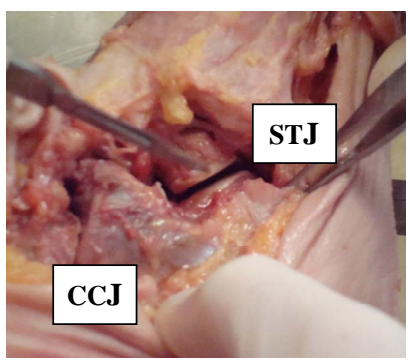

Figure 3. Cadaveric specimen demonstrating the STJ and CCJ.

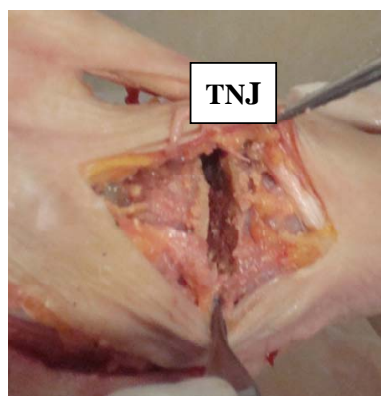

Figure 4. Cadaveric specimen demonstrating the TNJ.

placed perpendicular to each other at the tibial and ankle rings, one frontal plane wire and one medial face wire according to the safe zone placement by the Ilizarov method [2]. The wires were tensioned to $110 \mathrm{~kg}$ and tighten to the rings. Two crossing perpendicular smooth wires 
were also placed across the calcaneus and were also tensioned and tighten to the foot plate. As described by Talarico and Vito, one wire was placed through the talus and one through the cuboid and navicular. At this point, each cadaveric limb underwent two experimental trials.

\subsection{Trial \#1-Arched-Wire Technique}

One transosseous wire (talar wire) was placed through the talus from medial to lateral as described by Talarico and Vito, 2004. The placement is $1 \mathrm{~cm}$ anterior to medial malleolus. A second transosseous wire (midfoot wire) was placed medial to lateral from the body of the navicular exiting the body of the cuboid. At this point, the wires are arched according to the study. The talar wire is arched posteriorly and inferiorly and attached to the foot plate via appropriately sized posts (Figure 5). This is to simulate the force perpendicular to the talocalcaneal joint. The midfoot wire is also arched posteriorly and inferiorly to simulate perpendicular forces to the C-C and T-N joints and attached to the foot plate with appropriately sized posts (Figure 6). A third transosseous wire placed is the metatarsal wire from medial to lateral $1-1.5 \mathrm{~cm}$ from the base of the first metatarsal aiming to cross as many metatarsals possible exiting lateral $\left(4^{\text {th }}\right.$ or $5^{\text {th }}$ metatarsal base). It is built up with posts and attached to the foot plate in position.

The Tekscan 6900CR pressure sensors were placed in each of the three joints. The placement is consistent throughout the study. Sensor 1 is placed in the T-C joint; Sensor 2 is placed in the C-C joint and Sensor 3 is placed in the $\mathrm{T}-\mathrm{N}$ joint.

\subsubsection{Sequence $A$}

With all wires placed in the leg and the calcaneus, the talar and midfoot transosseous wires are arched back and

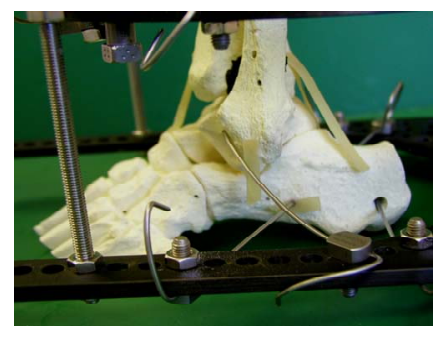

Figure 5. Demonstration of the orientation of the talar wire.

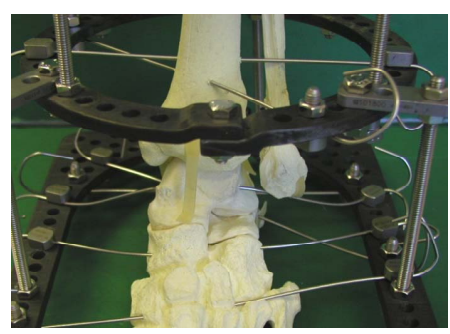

Figure 6. Demonstration of the orientation of the midfoot. secured to the foot plate. The midfoot wire is tightened on the lateral side and tensioned to $70 \mathrm{~kg}$ at the medial. It impacts on the C-C and T-N joints and the Sensors 2 and 3 measure the forces applied. The talar wire is then tightened on the medial side and tensioned to $70 \mathrm{~kg}$ at the lateral. It impacts on the T-C joint and the sensor 1 measures the forces applied. Observations were made at the effect of the T-C joint as the midfoot wire was tensioned and inherently, at the T-N and C-C joints as the talar wire was tensioned. The metatarsal wire was then tightened to one end and tensioned to $50 \mathrm{~kg}$ at the other end. It provides stability to the forefoot for weight bearing.

\subsubsection{Sequence $B$}

The sequence for tensioning was reversed and the forces measured. The tension was released at the talar and midfoot wires. The talar wire was first tensioned to $70 \mathrm{~kg}$, and then the midfoot wire was tensioned. Observations were made at the effects of the T-N and C-C joints as the talar wire was tensioned and inherently, the T-C joint as the midfoot wire was tensioned. Observations were recorded for the effects on all joints according to different tensions used, $50 \mathrm{~kg}$ and $70 \mathrm{~kg}$.

\subsubsection{Weight-Bearing Cycles}

Multiple trials with sequence A and B were performed and measurements recorded. Each of the four cadaveric limbs in sequence A and B were placed on the MTS 858 Mini Bionix testing system with the pressure sensors fixated in the joints. A femoral component was attached to the system and applied a $75 \mathrm{~kg}$ axial load to the limb to simulate the weight of an average person. The limb was then supplied with multiple weight-bearing cyclic periods. The activity of the three joints was observed and forces were measured (Figure 7).

\subsection{Trial \#2-No Arched-Wire Technique}

One cadaveric specimen was prepared in the exact method as Trail 1 and the transosseous wire placements were fashioned in the exact manner. The talar wire was placed and not arched; it was secured to the foot plate and built up with the appropriate post as it lay. The same procedure for the midfoot wire was performed. The metatarsal wire was placed as described in Trial 1. The Tekscan 6900CR pressure Sensors 1, 2, 3 were placed in the T-C, $\mathrm{C}-\mathrm{C}$ and $\mathrm{T}-\mathrm{N}$ joints respectively.

\subsubsection{Sequence $A$}

The cadaver limb underwent the same procedure without arching the wires as Trial 1 and the forces on the joints measured.

\subsubsection{Sequence $B$}

The cadaver limb underwent the same procedure without 


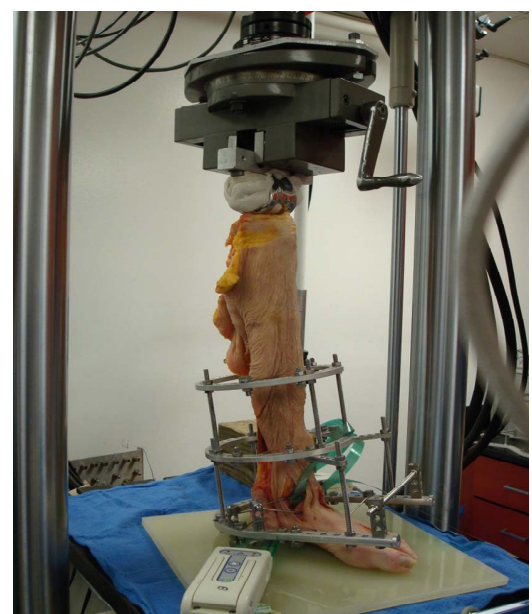

Figure 7. MTS 858 MiniBionix testing system simulating weightbearing cycles.

arching the wires as Trial 1 and the forces on the joints measured (Figure 8).

\subsubsection{Weight-Bearing Cycles}

Multiple trials with sequence A and B were performed and measurements recorded. The cadaveric limb in sequence A and B were placed on the MTS 858 MiniBionix testing system with the pressure sensors fixated in the joints. The same steps followed in Trial 1 were also performed in Trial 2.

\section{Results}

\subsection{Trial \#1-Arched-Wire Technique}

\subsubsection{Sequence $A$}

The midfoot wire was tightened on the lateral side and tensioned to 70 kilograms. The pressure sensors measured an average reading of $3.5 \mathrm{~N}$ which signifies adequate compression at the $\mathrm{C}-\mathrm{C}$ and $\mathrm{T}-\mathrm{N}$ joints. The talar wire was tightened on the medial side and tensioned to $70 \mathrm{~kg}$ on the lateral side. As the talar wire was being tensioned, it was noted that the pressure reading on the T-C joint increased but as the tension approached $70 \mathrm{~kg}$, the C-C and T-N joint forces were almost completely eliminated. When $70 \mathrm{~kg}$ of tension was placed on the talar wire at the T-C joint, the initial compression was lost at the C-C and $\mathrm{T}-\mathrm{N}$ joints. It was also noted that when the tension was released at the talar wire, the original pressure at the C-C and T-N joints returned. The procedure was attempted several times on the four cadavers with the same results (Table 1).

\subsubsection{Sequence $B$}

The sequence of tightening and tensioning the wire was reversed, starting with the talar wire at the T-C joint and then the midfoot wire at the $\mathrm{C}-\mathrm{C}$ and $\mathrm{T}-\mathrm{N}$ joints. This

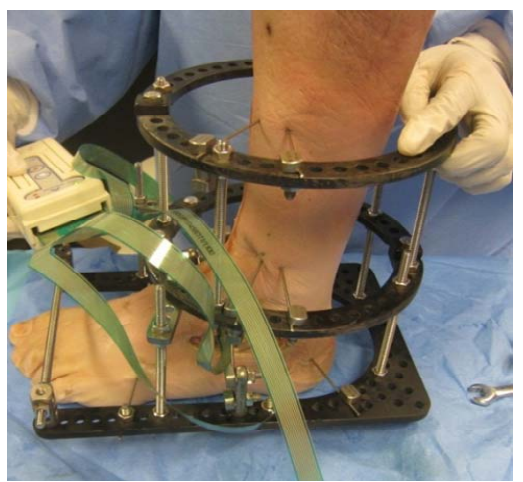

Figure 8. Demonstration of MTS 858 Minibionix $1 \times 11$ on weightbearing cadaveric limb.

time the T-C joint lost all of its pressure force reading when the $\mathrm{C}-\mathrm{C}$ and $\mathrm{T}-\mathrm{N}$ joints were being compressed.

At this point, it was decided that a third arch wire in the calcaneus may contribute some stability to the T-C joint and maintain the pressure. This third arched wire was a transosseus wire through the calcaneus. It was arched superiorly, to resist forces that could plantarflex the calcaneus. This wire was tightened and tensioned to the footplate with the utilization of appropriate hole posts. Now, the sequence was repeated several times. There was no change to the results. The third arched wire did not prove to hold the tension by preventing the calcaneus from plantar flexing and keeping it in one position (Table 2).

\subsubsection{Weight-Bearing Cycles}

The cadavers with external fixator and pressure sensors intact were mounted on to the MTS 858 MiniBionix II testing system. This machine was used to simulate weight bearing cycles. A weight of $200 \mathrm{~kg}$ was applied to the cadaver with the external fixator and the arched wires tensioned. The leg was then put through 200 cycles of simulated weight bearing. At this point, all pressure sensors were showing compression at the three joints. The five different cadavers were put through the same cycles and produced the same results.

\section{Discussion}

From this study it can be concluded that joint compression is not due to the arched wire technique but early weightbearing postoperatively. When weight bearing forces were applied to the leg with the external fixator and arched wires tensioned, compression at all three joints was achieved. The concept of external fixation with arched wire compression for triple arthrodesis has been applied for several years. The arched wires have been thought to provide compression at the three joints and aid in the fusion. The decrease of the pressure at the joints after sequential tensioning argues this point.

With regards to available literature there has been little 
Table 1. The effects of the forces on the three joints at end of Trial 1 (Arched) Sequence A.

\begin{tabular}{ccc}
\hline Pressure Sensor & Joint/Tensioned (70 kg) & Avg. Final Forces (N) \\
\hline Sensor 1 & T-C (Tensioned 2 $\left.{ }^{\text {nd }}\right)$ & $2.6-4.4$ \\
Sensor 2 & C-C & 0.5 \\
Sensor 3 & T-N & 0.4 \\
\hline
\end{tabular}

Table 2. The effects of the forces on the three joints at end Trial 1 (Arched) Sequence $B$.

\begin{tabular}{ccc}
\hline Pressure Sensor & Joint/Tensioned (70 kg) & Avg. Forces (N) \\
\hline Sensor 1 & T-C (Tensioned $\left.1^{\text {st }}\right)$ & 0.6 \\
Sensor 2 & C-C & $6.2-14.2$ \\
Sensor 3 & T-N & $6.2-7.6$ \\
\hline
\end{tabular}

Table 3. The effects of the forces on the three joints with weightbearing cycles.

\begin{tabular}{cccc}
\hline $\begin{array}{c}\text { Cadaver } \\
\text { Specimen }\end{array}$ & $\begin{array}{c}\text { Sensor 1 at T-C } \\
\text { Joint (N) }\end{array}$ & $\begin{array}{c}\text { Sensor 2 at C-C } \\
\text { Joint (N) }\end{array}$ & $\begin{array}{c}\text { Sensor 3 at T-N } \\
\text { Joint (N) }\end{array}$ \\
\hline Average & 78.8 & 44.5 & 18.4 \\
\hline
\end{tabular}

to no scientific evidence for triple arthrodesis with external fixation and weightbearing to increase compression. However, the clinical outcome resulted in adequate joint fusion. In regards to the fusion time, clinical results identified a decrease from the traditional 10 - 14 weeks to 8 10 weeks. Table 1 demonstrates that the effects of the forces on the three joints at the end of trial 1 with average final forces across the T-C, C-C and T-N respectively equaling $2.6-4.4 \mathrm{~N}, 0.5 \mathrm{~N}$ and $0.4 \mathrm{~N}$. The result identifies the T-C joint compressive forces to be significantly higher. While in Table 2, the results indicate in sequence B higher compressive forces across the arched wire compressing the $\mathrm{C}-\mathrm{C}$ and $\mathrm{T}-\mathrm{N}$ joints with ranges of $6.2-14.2 \mathrm{~N}$ for the C-C joint and $6.2-7.6 \mathrm{~N}$ for the T-N joint. Thus identifying constant compression forces applied to exclusively the arched wire being tensioned. Table 3 demonstrated that with weightbearing cycles the average forces on the T-C, C-C and T-N joints equaling $78.8 \mathrm{~N}, 44.5 \mathrm{~N}$ and $18.4 \mathrm{~N}$ respectively. This clearly demonstrates that weightbearing with the proper technique is directly proportional to compression leading to fusion.

The research gathered has lead to the development of the anatomical foot plate which is a promising tool in the future of foot and ankle reconstructive surgery. The anatomical foot plate has been used in performing a triple arthrodesis on 20 patients directly by the author. The foot plate has resulted in good to excellent clinical results, with further studies to be conducted.

Limitations of this study includes that there has been little to no research on the effects of weightbearing and triple arthrodesis. Further data needs to be obtained in order to perform comparative studies with triple arthrodesis using external fixation and early weightbearing. The versatility of the anatomical foot plate system has only been tested by the author.

In conclusion, early weightbearing alone provides compression at all 3 joints in a triple arthrodesis with external fixation.

\section{REFERENCES}

[1] E. W. Ryerson, “Arthrodesing Operations on the Feet,” Ournal of Bone and Joint Surgery, Vol. 5, No. 3, 1923, pp. 453471.

[2] M. H. El Ghawabi, "Centripetal Compression Triple Arthrodesis,” Acta Orthopaedica Scandinavica, Vol. 49, No. 3, 1978, pp. 306-309. doi:10.3109/17453677809005770

[3] G. R. Vito and L. M. Talarico, "Triple Arthrodesis Using External Ring Fixation and Arched-Wire Compression,” Journal of the American Podiatric Medical Association, Vol. 94, No. 1, 2004, pp. 12-21.

[4] P. D. Angus and H. R. Cowell, "Triple Arthrodesis: A Critical Long Term Review,” Journal of Bone and Joint Surgery, Vol. 68, No. 2, 1986, pp. 260-265.

[5] M. A. Catagni, "Atlas for the Insertion of Transosseous Wires and Half Pins,” Illizarov Method, Milan, 2002, pp. 31-46. 